\title{
THE THEORY OF THE NUTATION FOR A RIGID EARTH MODEL: CURRENT STATE OF THE SITUATION
}

\author{
J. SOUCHAY \\ Observatoire de Paris/DANOF, URA 1125 du CNRS, 61 Avenue de l'Observatoire, \\ F-75014, Paris; E-mail:"Souchay@obspm.fr" \\ AND \\ H. KINOSHITA \\ Tokyo National Astronomical Observatory, Ohsawa 2-21-1, Mitaka-Shi Tokyo 181, \\ Japan
}

\section{Introduction}

Whereas no particular attention was paid to the theory of the nutation for a rigid Earth model, for more than a decade after the adoption by the International Astronomical Union (IAU) of coefficients as calculated and listed by Kinoshita (1977), an increasing number of studies have been done in the recent years aiming to improve this theory. The improvement became necessary mainly because of the big parallel improvement of the VLBI observations itself, which leads to present determinations of some coefficients of nutation at the level of a few $10 \mu a s$. Therefore the amelioration of the theory of the nutation for a rigid Earth model can be divided in two aspects: one is to consider a smaller level of truncation of the coefficients of nutation; the other is to evaluate in the best way the coefficients already taken into account, particularly the leading coefficients which are typically those subject to the largest absolute differences.

As considering the first aspect, it seems that presently a level of truncation of $0.1 \mu a s$ for both $\Delta \psi \sin \varepsilon$ and $\Delta \varepsilon$ seems quite reasonable, for the global nutation obtained by summing the coefficients under this level does not exceed $\pm 3 \mu a s$, which is far below the level of the noise of the residuals between the most accurate VLBI observations and the theory for a non-rigid Earth model, whose the absolute amplitude is typically of the order of 0.1 mas, after fitting a few coefficients whose the modeling is not yet satisfactory, because of complex geophysical effects (see for instance Herring, 1991, Souchay et al., 1995).

As considering the second aspect, an agreement between the global nutations or the amplitudes of the coefficients as calculated by different authors, if not constituting an unquestionable proof of the validity of these coefficients, is a big step in order to insure this validity. Moreover, an additioning agreement between the analytical determination of the nutation and the determination obtained independently by the way of numerical integration would prove both that the analytical computations have been carried out properly, and that no contribution has been forgotten, at the level of truncation considered.

In Table 1, we summarize main of the various studies which have been done concerning the nutation for a rigid Earth model since 1985, with the possibility that the list be not complete. We mention when these studies resort to analytical computations, numerical integration, or both of them. We can notice the increasing number of works after a reconstruction of the theory by Kinoshita and Souchay (1990).

\section{Recent improvements}

Before the reconstruction of the theory of the nutation for a rigid Earth model by Kinoshita and Souchay (1990) the difference between the nutations given by numerical integration and the analytical tables (Kinoshita, 1977) exceeded 1 mas as was shown by Kubo and Fukushima (1988) 
TABLE 1. List of various studies dealing with rigid Earth nutation, from 1985. the symbol " $\mathrm{A}$ " (third column) is for "analytical", the symbol "N" for "numerical".

\begin{tabular}{|c|c|c|c|c|}
\hline AUTHOR(S) & YEAR & A & $\mathrm{N}$ & REMARKS AND CONTENTS \\
\hline Kubo and Fukushima & 1987 & & $\mathrm{x}$ & Comparison with tables Kinoshita (1977) \\
\hline Schastok et al. & 1988 & & $\mathrm{X}$ & Comparison with tables Kinoshita (1977) \\
\hline Zhu and Groten & 1989 & $\mathrm{X}$ & & $J_{2}, J_{3}$, second order, tables, fig. axis truncation: $0.01 \mathrm{mas}$ \\
\hline Schastok et al. & 1989 & & $\mathrm{X}$ & Comparison with tables Kinoshita and Souchay (1990) \\
\hline Kinoshita and Souchay & 1990 & $\mathrm{X}$ & & $\begin{array}{l}\text { Complete theory, } J_{2}, J_{3} \text {, second-order, planetary effects, } \\
\text { triaxiality, precession, } H_{d} \text {, tables } 3 \text { axes, truncation } 5 \mu a s\end{array}$ \\
\hline Souchay and Kinoshita & 1991 & & $\mathrm{x}$ & Comparison with tables Kinoshita and Souchay (1990) \\
\hline Hartmann and Soffel & 1994 & $\mathrm{X}$ & & Direct planetary effect from tidal porential \\
\hline Williams & 1994 & $\mathrm{X}$ & & Precession, $H_{d}$, tilt-effect, obliquity rate \\
\hline Hartmann et al. & 1995 & $\mathrm{X}$ & & $J_{3}$ effect from tidal potential \\
\hline Williams & 1995 & $\mathrm{X}$ & & Direct planetary effects, tables, truncation: $0.1 \mu a s$ \\
\hline Souchay and Kinoshita & 1996 & $\mathrm{X}$ & & $\begin{array}{l}\text { Corrections and new developments (I), lunisolar } J_{2} \text {, } \\
\text { indirect planetary effects, corrections, truncation: } 0.1 \mu \text { as }\end{array}$ \\
\hline Souchay and Kinoshita & 1997 & $\mathrm{X}$ & & $\begin{array}{r}\text { Corr. and new developments (II), obliquity rate, } J_{3} \text {, } \\
J_{4} \text {, direct planetary effects, truncation } 0.1 \mu a s\end{array}$ \\
\hline Hartmann and Soffel & 1997 & & & $\begin{array}{r}\text { Complete theory from tidal potential } \\
\text { (except diurnal and sub-diurnal), } J_{2}, J_{3}, J_{4} \\
\text { planetary effects, triaxiality, precession, } H_{d} \\
\text { tables } 3 \text { axes, truncation } 0.5 \mu a s\end{array}$ \\
\hline Roosbeek and Dehant & 1997 & $\mathrm{X}$ & $\mathrm{X}$ & $\begin{array}{r}\text { Complete theory (except diurnal and sub-diurnal), } J_{2}, J_{3} \\
\text { second order effects, planetary effects, triaxiality, precession, } H_{d} \\
\text { tables RDAN97, } 3 \text { axes, truncation } 0.1 \mu a s\end{array}$ \\
\hline Bretagnon et al. & 1997 & $\mathrm{X}$ & $\mathrm{X}$ & $\begin{array}{r}\text { Complete theory, } J_{2}, J_{3}, J_{4}, \text { triaxiality, } \\
C_{3, i}, C_{4_{1}, i}, C_{5, i}, \text { planetary effects, precession, } H_{d} \\
\text { tables, } 3 \text { axes, truncation }<0.01 \mu a s\end{array}$ \\
\hline Folgueira et al. & 1997 & $\mathrm{X}$ & & Nutations from $C_{3, i}, S_{3, i}$ \\
\hline Folgueira et al. & 1997 & $\mathrm{X}$ & & Nutations from $C_{4, i}, S_{4, i}$ \\
\hline Souchay et al. & 1997 & $\mathrm{X}$ & & $\begin{array}{r}\text { Corr. and new developments (III), complete theory } \\
J_{2}, J_{3}, J_{4}, C_{3, i}, C_{4, i} \text {, second-order, } \\
\text { planetary effects, triaxiality, precession, } H_{d}, \\
\text { tables "REN-2000", } 3 \text { axes, trunciation } 0.1 \mu a s\end{array}$ \\
\hline Souchay & 1997 & & $\mathrm{X}$ & $\begin{array}{r}\text { Numerical integration: comparisons with tables REN-2000, } \\
\text { comparisons inter-tables : REN-2000 (Souchay et al., 1997), } \\
\text { RDAN97 (Roosbeek and Dehant, 1997) } \\
\text { SMART97 (Bretagnon et al., 1997) }\end{array}$ \\
\hline
\end{tabular}

as well as by Schastok et al. (1987). Clearly some big discrepancies occurred for the terms with argument $\Omega(18.6 \mathrm{yr})$ and $2 \Omega(9.3 \mathrm{yr})$ both in longitude and in obliquity.

The reason is that two kinds of effects were not taken into account in the analytical tables. One is the spin-orbit coupling effect, firstly pointed out by Kubo (1982), which consists in an interaction between the rotation of the Earth and the orbital motion of the Moon, and the other is the crossednutation effect. They were both calculated simultaneously by Zhu and Groten (1989) as well as by 
Kinoshita and Souchay (1990), in the frame of the Hamiltonian theory for which the Earth-Moon system is considered as a whole (the results between these authors were quite in accordance). They are at the origin of the considerable reduction of the differences between the nutations as calculated by numerical integration and those calculated starting from the analytical tables (Schastok et al, 1989; Souchay and Kinoshita, 1991). Nevertheless some small differences remained for the leading components with period $18.6 \mathrm{yr}, 9.3 \mathrm{yr}$ semi-annual and fortnightly, which suggested that some corrections remained to be done in the analytical calculations.

A significant one was pointed out by Williams (1994), which consisted in including out-of-phase components for the nutations with arguments $\Omega$ and $2 \Omega$ both for $\Delta \psi$ and $\Delta \varepsilon$, due to the planetary tilt-over effect (the Moon's orbit is precessing with respect to an axis which is slightly shifted with respect to the axis of the ecliptic). Recently a significant additioning out-of-phase component in $\Delta \psi$ for the $\Omega$ term has been noticed by Bretagnon et al. (1997). The related ratio $\rho$ for this out-of-phase component to the in-phase one has been evaluated by Souchay et al. (1997) analytically with the following formula: $\rho=-\frac{2 \dot{\varepsilon}}{\dot{\Omega}} \tan 2 \varepsilon_{0}$ for $\Delta \psi$, which gives a 0.248 mas amount, and matches perfectly the difference of 0.250 mas found by Bretagnon et al. (1997). Roosbeek and Dehant (1997) made also a detailed analysis of the contributions to the out-of-phase components for the $\Omega$ term, which are quite in agreement with Bretagnon et al. (1997) and Souchay et al. (1997). Moreover Souchay et al. (1997) recalculated accurately the second-order tilt-effect and crossed-nutation effects at the level of $0.1 \mu a \mathrm{~s}$ instead of $5 \mu a \mathrm{~s}$ (Kinoshita and Souchay, 1990) and thus showed that they are at the origin of significant amplitudes around the semi-annual and fortnightly frequencies.

Concerning the nutations due to second-order harmonics of the geopotential, Kinoshita (1977) showed that the triaxiality of the Earth, characterized by the $C_{2,2}$ and $S_{2,2}$ tesseral harmonics, give birth to several coefficients at the level of 0.01 mas, with nearly semi-diurnal period. They were calculated up to $5 \mu a s$ by Kinoshita and Souchay (1990) who calculated also the coefficients due the influence of the geopotential $J_{3}$ which could no more be neglected at this level of truncation, with a leading coefficient at the level of 0.1 mas. Souchay and Kinoshita (1997) recalculated these two kinds of nutations up to $0.1 \mu a s$, with quite a good agreement concerning the second effect with the results of Hartmann et al. (1996). Bretagnon et al. (1997) showed that the $C_{3, i}, S_{3, i}, C_{4, i}, S_{4, i}$ harmonics produce also a significant number of coefficients up to $0.1 \mu \mathrm{as}$ with a leading one closed to 40uas. Their period is close to diurnal, semi-diurnal or 1/3 diurnal. Folgueira et al. (1997a, 1997b) found results in total agreement with Bretagnon et al. (1997) for these coefficients, but by the way of a theory based on the Hamiltonian canonical equations, thus completing the series related to this theory at the level of $0.1 \mu a s$ (Souchay and Kinoshita, 1996; Souchay and Kinoshita, 1997; Souchay et al., 1997). They showed that the Oppolzer terms, which make the difference between the angular momentum axis and the figure axis, are much larger than the respective coefficients for the angular momentum axis, which is a specific property of these terms.

A study of the direct action of the planets on the nutation have been done firstly by Vondrak (1983), then by Kinoshita and Souchay (1990), Hartmann and Soffel (1994), Williams (1995), Souchay and Kinoshita (1997). The agreement between these three last studies when comparing the coefficients up to $0.1 \mu$ as is remarkable, with only a few differences exceeding this level of truncation (Souchay and Kinoshita, 1997).

\section{Comparison between global theories and numerical integrations}

We can notice recently the accomplishment of four works aiming to the construction of complete or quasi-complete series of the nutation for a rigid-Earth model. They have been done and described precisely by Hartmann and Soffel (1997), Bretagnon et al. (1997), Roosbeek and Dehant (1997) and Souchay et al. (1997). The series are quoted respectively as HS97, SMART97, RDAN97 and REN2000. In Table 2 we summarize some of the main particularities of the series, for which we can remark fundamental differences in the way they have been constructed. For instance the series REN-2000 (Souchay et al. , 1997) are computed starting from Hamiltonian canonical equations (Kinoshita, 1977; Kinoshita and Souchay, 1990) in opposite to the other series for which the Dynamical Euler's equations are used. Moreover Bretagnon et al. (1997) for the establishment of their series SMART97 do not separate the different contributions to the nutation, which is not the case of the other authors who were concerned about making a detailed analysis of each of the effects involved. 
TABLE 2. Comparison between 4 recent rigid Earth nutation theories.

\begin{tabular}{|c|c|c|c|c|}
\hline Item & $\begin{array}{l}\text { Hartmann } \\
\text { and Soffel } \\
(1997)\end{array}$ & $\begin{array}{c}\text { Roosbeek } \\
\text { and Dehant } \\
(1997)\end{array}$ & $\begin{array}{r}\text { Bretagnon et } \\
\text { et al. } \\
(1997)\end{array}$ & $\begin{array}{r}\text { Souchay } \\
\text { et al. } \\
(1997)\end{array}$ \\
\hline Series name & $\mathrm{HS} 97$ & RDAN97 & SMART 97 & REN-2000 \\
\hline Basic equations & Tidal potential & Euler's. Eq. & Euler's Eq. & Hamiltonian \\
\hline Basic ref. syst. & Fixed & Fixed & Fixed & Moving \\
\hline Way of study & each contribution & each contribution & global & each contribution \\
\hline$J_{2}$ & - & - & - & - \\
\hline$J_{3}$ & - & - & - & - \\
\hline$J_{4}$ & - & - & - & - \\
\hline$C_{2,2}, S_{2,2}$ & - & - & - & - \\
\hline$C_{3,1}, S_{3,1}$ & no & no & - & - \\
\hline$C_{4,1}, S_{4,1}$ & no & no & - & - \\
\hline$J_{2}$ Planetary & - & - & - & - \\
\hline Truncation limit & $0.5 \mu a s$ & $0.1 \mu a s$ & $<0.01 \mu a s$ & $0.1 \mu a s$ \\
\hline Number of terms & & $\approx 1500$ & 10000 & $\approx 1500$ \\
\hline Tables & 3 axes & 3 axes & 3 axes & 3 axes \\
\hline $\begin{array}{l}\text { Comparison with } \\
\text { Numerical. Int. }\end{array}$ & no & 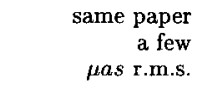 & $\begin{array}{r}\text { same paper } \\
\text { a few } \\
\text { Has r.m.s. }\end{array}$ & $\begin{array}{l}\text { this paper } \\
\text { a few } \\
\text { Has r.m.s. }\end{array}$ \\
\hline $\begin{array}{l}\text { Dyn. ellip. } \\
H_{d} \times 10^{3}\end{array}$ & 3.273792489 & 3.2737670 & 3.2737671 & 3.2737548 \\
\hline
\end{tabular}

We can also remark that only two series, SMART97 (Bretagnon et al., 1997) and REN-2000 (Souchay et al., 1997) can be considered as really complete up to $0.1 \mu a \mathrm{~s}$, for they are the only ones to include the effects due to the second-order harmonics of the geopotential $C_{3, i}, S_{3, i}(i=1,2,3)$ and $C_{4, i}, S_{4, i}(i=1,2,3,4)$, which are characterized by quasi-diurnal or sub-diurnal coefficients with about thirty of them having an absolute amplitude exceeding $1 \mu a s$ (Bretagnon et al., 1997; Folgueira et al., 1997) for $\Delta \psi$. The work of Hartmann and Soffel seems to be the more accurate and complete done so far in the attempt to make the relationships between the coefficients of the series of the tidal potential (Hartmann and Wenzel, 1995), and the coefficients of the nutation. Unfortunately, their method is not well suitable for the determination of low-frequency nutations because the uncertainty in the determination of the coefficients grows with their period, as was already noticed by Souchay (1993) when comparing analytical nutations.

The comparison with a numerical integration has been done at the same time as the analytical computations of the coefficients by Roosbeek and Dehant (1997) for the series RDAN97 and by Bretagnon et al. (1997), for the series SMART97. It is satisfactory at the level of a few microarcseconds in the two cases, but the residuals for a short-time span are particularly small in the second study, which is a proof that the analytical computations have been done with a very good internal accuracy. Moreover a specific study of the comparison of the series REN-2000 both with numerical integration and with the series RDAN97 and SMART97 has been done independently by Souchay (1997). The results found in this last study show that the residuals between analytical nutation from the series REN-2000 and numerical integration have a $3.2 \mu a s$ r.m.s and a $5.5 \mu a s$ r.m.s. respectively for $\Delta \psi \sin \varepsilon$ and $\Delta \varepsilon$ for a 134 years time span. The residuals are much noisy at high frequency than those obtained by Bretagnon et al. (1997), but this might be only due to the truncation of the series. REN-2000 truncated at $0.1 \mu \mathrm{as}$ contain no more than 1537 terms and 1192 terms respectively for $\Delta \psi$ and $\Delta \varepsilon$, whereas the series SMART97 contain several thousands of 
coefficients.

Anyway the agreement between the three series can be considered as very satisfactory. For instance Souchay (1997) showed that the residuals are about $5 \mu a s$ for $\Delta \psi \sin \varepsilon$ and $\Delta \varepsilon$ still for a 134 years time span, when comparing the global nutations of the figure axis from J2000.0, as calculated from REN-2000 and SMART97, after fitting only the $18.6 \mathrm{yr}$ leading component. Moreover he showed that the absolute difference concerning this term, essentially due to different values adopted to the dynamical ellipticity of the Earth $H_{d}$, is less than $10 \mu a s$ when adopting the same value of $H_{d}$. The residuals between the nutations as computed from RDAN97 and REN-2000, although a little less convincing, are still at the level of a few microarcseconds r.m.s.

Therefore we can conclude that a big step has been done recently in the frame of rigid Earth nutation theory, and that the coherence between the various studies is established, thus leading to the possibility of a definitive adoption of series at the level of truncation of $0.1 \mu a s$.

\section{References}

Bretagnon, P., Rocher, P. and Simon, J.L. (1997) Astron. Astrophys., 319, pp. 305.

Folgueira, M., Souchay, J. and Kinoshita, H. (1997) Celest. Mechanics, submitted.

Folgueira, M., Souchay, J. and Kinoshita, H. (1997) Celest. Mechanics, to be submitted.

Hartmann, T. and Soffel, M. (1994) Astron. J., 108, pp. 1115.

Hartmann, T., Williams, J.G. and Soffel, M.. (1996) Astr. J., 111, pp. 1400.

Hartmann, T. and Wenzel, H.G. (1995) Geophys. Res. Letters, 22, pp. 3553.

Hartmann, T. and Soffel, M. (1997) Celest. Mech., submitted.

Herring, T. (1991) in: Proceedings of the 127th. Colloquium of the International Astronomical Union, Reference Systems, ed. J.A. Hughes, C.A. Smith, G.H. Kaplan, U.S. Naval Observatory, Washington.

Kinoshita, H. (1977) Celest. Mech, 15, pp. 277.

Kinoshita, H. and Souchay, J. (1990) Celest. Mech., 48, pp. 187.

Kubo, T. (1982) Celest. Mech., 26, pp. 96.

Kubo, Y. and Fukushima, T. (1988) in: The Earth's Rotation and Reference Frames for Geodesy and geodynamics, ed. A.K. Babcock and G.A. Wilkins, pp. 331 .

Roosbeek, F. and Dehant, V. (1997) Celest. Mechanics, submitted.

Schastok, J., Soffel, M. and Ruder, H. (1987) in: Proc. IUGG symp. U4, Geophysical Monograph, 59, pp. 17-20.

Schastok, J., Soffel, M. and Ruder, H. (1989) Celest. Mech., 47, pp. 219.

Souchay, J. and Kinoshita, H. (1991) Celest. Mech., 52, 45

Souchay, J. (1993) Astron. Astrophys., 276, pp. 266.

Souchay, J., Feissel, M., Bizouard, C., Capitaine, N. and Bougeard, M. (1995) Astron. Astrophys., 299, pp. 277.

Souchay, J. and Kinoshita, H. (1996) Astron. Astrophys., 312, pp. 1017.

Souchay, J. and Kinoshita, H. (1997) Astron. Astrophys., 318, pp. 639.

Souchay, J., Loysel, B., Kinoshita, H. and Folgueira, M. (1997) Astron. Astrophys., submitted.

Souchay, J. (1997) Astron. Jour., submitted.

Vondrak, J. (1983) Bull. Astr. Inst. Czechosl., 33, pp. 26.

Williams, J.G. (1994) Astron. J., 108, pp. 711.

Williams, J.G. (1995) Astron. J., 110, pp. 1420.

Zhu, S.Y. and Groten, E. (1989) Astron. J., 98, pp. 1104. 